VLBI POSITIONS OF EIGHT STELLAR SYSTEMS

A.E. Niell (1), J.-F. Lestrade $(2,3)$, R. A. Preston (2), R.L. Mutel (4), and R. B. Phillips (1)

(1) Haystack Observatory, Westford, MA 01886

(2) Jet Propulsion Laboratory, Pasadena, CA 91109

(3) Bureau des Longitudes, Paris, France F-75014

(4) University of Iowa, Iowa City, IA 52242

ABSTRACT. Radio positions of eight stellar systems have been derived with accuracies of 3 to 300 milliarcseconds from MkIII VLBI observations conducted with multi-station arrays. The best accuracy was obtained during a relatively strong outburst of the RS CVn system HR 5110. The epoch J2000.0 positions are obtained in the Jet Propulsion Laboratory VLBI reference frame of extragalactic radio sources.

1. Radio star astrometry and the Hipparcos Satellite

Observations of optically bright stars using VLBI are being made to determine accurate positions (uncertainties of a few milliarcseconds) and proper motions for about 15 objects well distributed over the sky. The extragalactic radio sources which form the reference frame for these positions will then provide an inertial frame for the optical positions of 100,000 stars determined by the Hipparcos satellite. The accuracy expected for the satellite observations is 2 mas in position and $2 \mathrm{mas} / \mathrm{yr}$ in proper motion.

\title{
2. Observations and Results
}

Survey work has shown that only the class of stars called RS Canum Venaticorum and other close binary systems (e.g. Algol, CYG X-1, LSI61 303) are at times sufficiently strong and compact in their radio emission to be useful for the astrometric requirements. During three sessions in 1983, using arrays of 5 or 6 antennas, we determined positions for eight stars with formal uncertainties of 0.003 to $0 ! 3$ (see tables 1 and 2). No correction has been made for proper motion or parallax. The precision was limited primarily by the strengths of the sources, which ranged from 9 to $400 \mathrm{mJy}$ at the times of the observations.

We have compared these results with radio positions obtained with the VLA and with optical positions after transforming the VLA and 327

M. J. Reid and J. M. Moran (eds.), The Impact of VLBI on Astrophysics and Geophysics, 327-328.

(C) 1988 by the IAU. 
optical positions from epoch B1950.0 to J2000.0. For 5 sources the mean (and rms) of the differences (VLA-VLBI) are 0!00 (0!06) and -0!06 (0"06) in right ascension and declination, respectively. For seven stars, excluding Algol, the mean (and rms) of the differences with the optical (OPTICAL-VLBI) are 0!03 (0!08) and -0!01 (0!09). The good agreement with the optical in right ascension is fortuitous since the origin of right ascension for the radio frame has an uncertainty of 0.2 with respect to the optical (Hazard et al., 1971). However, it does indicate that the JPL VLBI radio reference frame is aligned with the preliminary FK5 frame at a level of better than 0 "1. This uncertainty is limited by the systematic errors over the sky of 0"1 to 0.2 in the optical positions.

Table 1. Dates of observations

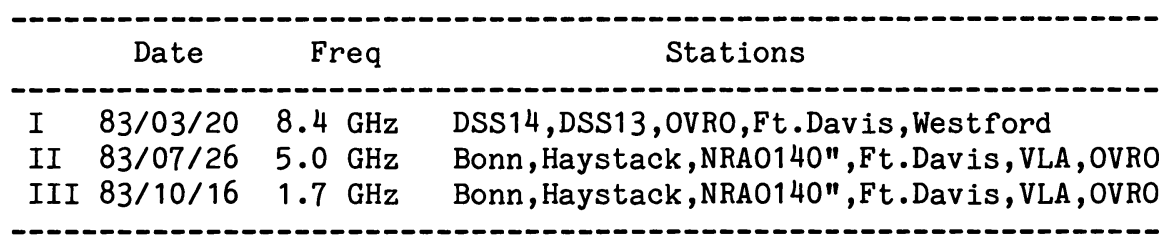

Table 2. Positions of eight radio stars. No correction has been made for parallax or proper motion.

\begin{tabular}{|c|c|c|c|c|c|c|c|c|c|c|}
\hline \multirow[t]{2}{*}{ Star } & \multicolumn{2}{|c|}{ Session } & \multicolumn{3}{|c|}{ Right Ascension } & \multicolumn{3}{|c|}{ Declination } & \multicolumn{2}{|c|}{$\begin{array}{l}\text { Flux } \\
\text { Density }\end{array}$} \\
\hline & & $\mathrm{hr}$ & $\mathrm{mn}$ & sec & & $\mathrm{dg}$ & $\mathrm{mn}$ & $\mathrm{sec}$ & & $(\mathrm{mJy})$ \\
\hline I61 303 & III & 02 & 40 & $31.686+1$ & -0.008 & 61 & 13 & 45.56 & $+/-0.07$ & 40 \\
\hline gol & II & 03 & 08 & 10.1308 & 0.0003 & 40 & 57 & 20.359 & 0.005 & 45 \\
\hline UX Ari & II & 03 & 26 & 35.3375 & 0.0002 & 28 & 42 & 56.026 & 0.005 & 145 \\
\hline HR 1099 & I & 03 & 36 & 240 & 0.0002 & 00 & 35 & 18.66 & 0.02 & 400 \\
\hline HR 5110 & II & 13 & 34 & 893 & 0.0002 & 37 & 10 & 56.859 & 0.003 & 165 \\
\hline SigCrB & II & 16 & 14 & 41.2011 & 0.0007 & 33 & 51 & 32.47 & 0.01 & 16 \\
\hline CygX -1 & II & 19 & 58 & 21.6804 & 0.0004 & 35 & 12 & 05.887 & 0.009 & 15 \\
\hline SZ Psc & II & 23 & 13 & 23.7645 & 0.0005 & 02 & 40 & 31.31 & 0.04 & 38 \\
\hline
\end{tabular}

\section{REFERENCES:}

Hazard,C, Sutton, J., Argue, A.N., Kenworthy, C.M., Morrison, L.V., and Murray, C.A., 1971, Nature, 233,89. 